\title{
Threshold $e^{-} p \rightarrow n v_{e}$ scattering and the electron neutrino mass
}

\author{
Jacek Ciborowski ${ }^{1, a}$ and Jakub Rembieliński ${ }^{2}$ \\ ${ }^{1}$ University of Warsaw, Faculty of Physics, L. Pasteura 5, 02-093 Warsaw, Poland \\ ${ }^{2}$ University of Łódź, Faculty of Physics and Applied Informatics, Pomorska 149/153, 90-236 Łódź, Poland
}

\begin{abstract}
The most precise measurement of the electron neutrino mass has been obtained from the shape of the electron energy spectrum near the endpoint in tritium decay. The Mainz and Troitsk experiments indicated an excess instead of expected depletion of counts in that region. Results derived from such measurements are subject to numerous atomic corrections which are absent in the scattering $e^{-} p \rightarrow n v_{e}$. This new idea is presented in the article, with its advantages and difficulties, and is compared to the method of tritium decay.
\end{abstract}

\section{Introduction}

In this article a new idea, regarding the issue of measuring the electron neutrino mass, is presented and its main advantages as well as difficulties discussed in short. Since it is practically unachievable to measure the velocity of the neutrino by a time-of-flight method, and given the energy - its mass, the only avialable methodology must rely on studying a process in which a finite neutrino mass would lead to a modification of kinematics in a particular region of the phase space. To date, the most fruitful method, which allowed to determine an upper limit on the electron neutrino mass with $\mathrm{eV}$-precision, was the study of the endpoint region of the electron kinetic energy spectrum in a $\beta$-decay of tritium. In this decay, ${ }^{3} \mathrm{H} \rightarrow{ }^{3} \mathrm{He} e^{-} \bar{v}_{e}$, the maximum electron kinetic energy is rather low (18.6 keV); moreover, the decay is characterised by an acceptable half-life time of 12.3 years. A finite neutrino mass would manifest as a depletion of counts, compared to the case of the massless neutrino, in the electron kinetic energy distribution near the endpoint. The latest results for the limit on the electron neutrino mass have been derived over a decade ago from data collected in two $\mathrm{eV}$-precision experiments: at Troitsk (Russia) [1-4] $\left(m_{v}<2.05 \mathrm{eV}\right.$ at 95\% c.1.) and Mainz (Germany) [5, 6], [7] ( $m_{\mu}^{2}=-0.6 \pm 2.2$ (stat) \pm 2.1(syst) $\mathrm{eV}^{2}$ or $m_{v}<2.3 \mathrm{eV}$ at $95 \%$ c.1.). A new generation experiment, KATRIN, is currently at the commissioning stage, aiming at a sub-eV precision constraint or measurement of the electron neutrino mass [8]. A number of other experiments, for the time being less precise, are also currently in progress, including bolometric experiments MIBETA [9] $\left(m_{\mu}^{2}=-141 \pm 211\right.$ (stat) \pm 90 (syst) $\left.\mathrm{eV}^{2}\right)$, MANU [10] and MARE experiment [11] to measure the endpoint in the $\beta$ decay of ${ }^{187} \mathrm{Re}$ and the ECHo experiment [12] making use of the electron capture in ${ }^{163}$ Ho. The accuracy of determining the neutrino mass in a $\beta$ decay is limited by the precision of measuring the electron energy. A new related method has recently been demonstrated using a radio-frequency spectrometer for single electrons with the operation principle based on the relativistic shift in the cyclotron frequency [13], opening a new

a e-mail: cib@fuw.edu.pl 
perspective for the electron neutrino mass determination. It is a common feature of the results for $m_{v}^{2}$ that they come out systematically negative in most measurements. This feature is a sign either of an effect commonly unaccounted for in the data analysis or, if physical, a need of a new concept of the neutrino [14]. Therefore a measurement using a yet different technique might be of importance in this respect.

\section{Threshold $e^{-} p \rightarrow n v_{e}$ scattering}

We have considered another process, in which the fact of a finite neutrino mass would be reflected in its kinematics: $e^{-} p \rightarrow n v_{e}$ (a charged current process, CC, compared to a neutral current elastic $e^{-} p$ scattering). Again, a depletion of counts, compared to the massless neutrino case, in the energy dependence of the cross section for this channel close above the threshold is expected. The above elementary process has been chosen chosen by us for simplicity but it is understood that, in general, one can consider a process: $e^{-} A \rightarrow B v_{e}$, where $A$ and $B$ denote nuclei (although it has been known from the tritium decay experience that dealing with nuclei involves numerous nuclear and atomic corrections).

The differential cross section for the process $e^{-} p \rightarrow n v_{e}$, as a function of the electron-proton c.m.s. energy, $W$, takes the following form:

$$
\frac{d \sigma}{d \Omega}=\frac{|\mathcal{M}|^{2}}{256 \pi^{2}} \frac{1}{W^{2}} \sqrt{\frac{\left(W^{2}-m_{n}^{2}+m_{v}^{2}\right)^{2}-4 m_{v}^{2} W^{2}}{\left(W^{2}-m_{p}^{2}-m_{e}^{2}\right)^{2}-4 m_{p}^{2} m_{e}^{2}}},
$$

where $\mathcal{M}$ denotes the matrix element and $m_{n}, m_{p}, m_{e}$ and $m_{v}$ - the neutron, proton, electron and neutrino masses, respectively. The leading kinematical dependence of the cross section is contained in the $W^{2}$-dependent phase space term since the energy dependence of the matrix element is not strong near the threshold. Therefore we focus on the kinematical ( $W$-dependent) term in (1) in further considerations which is sufficient for illustrating of the underlying idea presented in this paper. The dependence of the cross section in terms of the c.m.s. energy, $W$, near the threshold for the process $e^{-} p \rightarrow n v_{e}$ is shown in Fig. 1 for the massive and massless neutrino. In this particular calculation

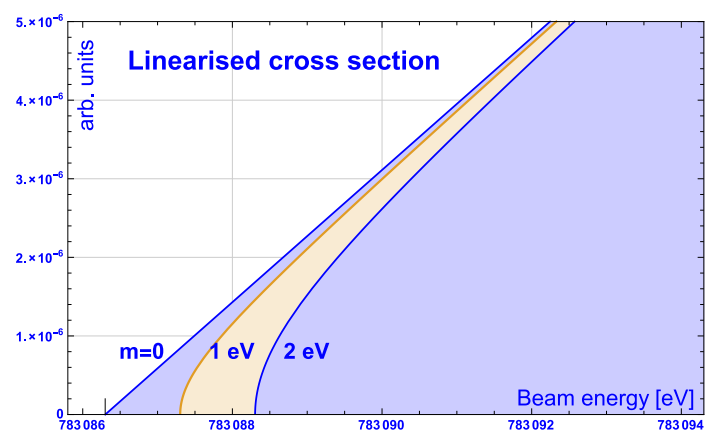

Figure 1. Energy dependence of the cross section for the process $e p \rightarrow n v$ near the threshold for a massive $\left(m_{v}=1,2 \mathrm{eV}\right)$ and massless electron neutrino.

we used the following values for the masses of the electron, the proton and the neutron [15]: $m_{e}=$ $\left.0.510998928 \mathrm{MeV}, m_{p}=938.272046\right) \mathrm{MeV}$ and $m_{n}=939.565378 \mathrm{MeV}$, respectively. The threshold value of the centre-of-mass energy for the massive and massless neutrino reads: $W_{t h}^{m}=m_{n}+m_{v}$ and 
$W_{t h}^{0}=m_{n}$, respectively. If the neutrino were massive, the threshold energy would be shifted towards higher values by $m_{v}$ and one would observe a depletion of counts compared to the massless case, as shown in the figure. The measurement would have to involve a beam energy scan, covering a region from below to above the threshold and the possible neutrino mass could be determined by fitting the functional dependence (1).

There are several difficulties associated with the method presented in this article.

- A low energy dispersion beam (order of eV), within an appropriate energy range below and above the energy threshold, is required since the accuracy of measuring the neutrino mass depends strongly on this parameter; the threshold kinetic energy of an electron beam on a stationary proton target amounts to approx. $0.783 \mathrm{MeV}$. The dispersion should be of order $10^{-6}$ of the beam energy which would be a challenging task to achieve in the case of the discussed process;

- A dominating elastic background is present, $e^{-} p \rightarrow e^{-} p$, requiring an efficient procedure of rejection; $\sigma_{C C} / \sigma_{e l} \approx O\left(10^{-12}\right)$, depending on acceptance, where $\sigma_{C C}$ denotes the cross section for the studied process;

- Low $C C$ cross section near threshold, $\sigma_{C C} \approx G_{F}^{2} s \approx O\left(10^{-4}\right) \mathrm{nb}$, and in consequence low event rates;

- Detection of a low energy neutron in the final state via a secondary process, like ${ }^{2} D$ formation with an accompanying $\gamma(2.22 \mathrm{MeV})$.

A comparison of the two methods: tritium decay and the threshold scattering, is summarised in table 1 .

Table 1. Comparison of key features characterising the two methods, tritium decay and threshold $e^{-} p$ scattering, of measuring the electron neutrino mass.

\begin{tabular}{lll}
\hline Process & tritium decay & $e^{-} p \rightarrow n v_{e}$ \\
\hline Region of study & below endpoint & above threshold \\
Effect searched in count rates & depletion & depletion \\
Method & final state $e^{-}$energy measurement & $\bullet e^{-}$beam energy setting (scan) \\
& & $\bullet$ final state neutron detection \\
Precision related device & high precision $e^{-}$spectrometer & low dispersion $e^{-}$beam line \\
Device dimension, scale & 3D, very large & 1D, moderate \\
Background processes & none & elastic $e^{-} p$ scattering \\
Experience status & decades of practice & intention stage \\
\hline
\end{tabular}

In summary, we have shown that the energy dependence of the cross section for the process $e^{-} p \rightarrow$ $n v_{e}$ above threshold is sensitive to a possibly non-zero electron neutrino mass - an effect quantitatively similar to that near the endpoint of the electron energy spectrum in tritium decay. The new method requires further elaboration and a feasibility study while the main difficulties, which can be listed a priori, are: order of $\mathrm{eV}$ electron beam energy setting in the threshold range, abundant elastic $e^{-} p$ scattering background, low counting rates of the measured process and necessity of detecting slow neutrons.

This work was partially supported from the funds of the National Science Centre, contract DEC2012/06/M/ST2/00430.

\section{References}

[1] A. Belesev et al., JETP Lett. 97, 67 (2013) 
[2] A. Belesev et al., J. Phys. G41, 015001 (2014)

[3] V. M. Lobashev et al., Phys. Lett. B460, 227 (1999)

[4] V. N. Aseev et al., Phys. Rev. D84, 112003 (2011)

[5] Ch. Kraus et al., Eur. Phys. J. C73, 2323 (2013)

[6] Ch. Kraus et al., Eur. Phys. J. C40, 447 (2005)

[7] E. W. Otten and Ch. Weinheimer, Rep. Prog. Phys. 71086201 (2008)

[8] Ch. Weinheimer for the KATRIN collaboration, Progress in Particle and Nuclear Physics, 48, 141 (2002); http://www.katrin.kit.edu/ and references therein

[9] M. Sisti et al., NIM A 520 (2004) 125

[10] F. Gatti, Nucl. Phys. B91 293 (2001)

[11] S. Sangiorgio et al. Prog. Part. Nuc. Phys. 57, 68-70 (2006); A. Nucciotti et al., Nuclear Physics B (Proc. Suppl.) 229-232 155-159 (2012)

[12] L. Gastaldo et al., J. Low Temp. Phys. 176, 876 (2014); S. Eliseev et al., Phys. Rev. Lett. 115, 062501 (2015)

[13] D. M. Asner et al., Phys. Rev. Lett. 114, 162501 (2015)

[14] J. Ciborowski and J. Rembieliński, Eur. Phys. J. C8 157 (1999)

[15] Review of Particle Physics, K. Olive et al., Particle Data Group, Chin. Phys. C38 0900001 (2014); pdg.lbl.gov 